\title{
ON A CONVERGENCE THEOREM FOR THE LAGRANGE INTERPOLATION POLYNOMIALS
}

\section{G. GRÜNWALD}

The unique polynomial of degree $(n-1)$ assuming the values $f\left(x_{1}\right), \cdots, f\left(x_{n}\right)$ at the abscissas $x_{1}, x_{2}, \cdots, x_{n}$, respectively, is given by the Lagrange interpolation formula

$$
L_{n}(f)=f\left(x_{1}\right) l_{1}(x)+f\left(x_{2}\right) l_{2}(x)+\cdots+f\left(x_{n}\right) l_{n}(x) .
$$

Here

$$
l_{k}(x)=\frac{\omega(x)}{\omega^{\prime}\left(x_{k}\right)\left(x-x_{k}\right)}, \quad k=1,2, \cdots, n
$$

(fundamental polynomials of the Lagrange interpolation), and the polynomial $\omega(x)$ is defined by

$$
\omega(x)=c\left(x-x_{1}\right)\left(x-x_{2}\right) \cdots\left(x-x_{n}\right),
$$

where $c$ denotes an arbitrary constant not equal to zero. It is known and easy to verify that

$$
l_{1}(x)+l_{2}(x)+\cdots+l_{n}(x) \equiv 1 .
$$

In the Lagrange interpolation formula let

$$
x_{k}=x_{k}^{(n)}=\cos (2 k-1) \pi / 2 n=\cos \theta_{k}^{(n)}
$$

which implies

$$
\omega(x)=T_{n}(x)=\cos (n \operatorname{arc} \cos x)=\cos n \theta, \quad \cos \theta=x
$$

(Tchebyscheff polynomial). In this case we have

and

$$
\begin{aligned}
l_{k}(x)=l_{k}[\theta]=(-1)^{k+1} \frac{\cos n \theta \sin \theta_{k}^{(n)}}{n\left(\cos \theta-\cos \theta_{k}^{(n)}\right)}, \\
\quad k=1,2, \cdots, n ; x=\cos \theta ;
\end{aligned}
$$

(8) $\quad L_{n}(f)=L_{n}[f ; \theta]=\sum_{k=1}^{n} f\left(\cos \theta_{k}^{(n)}\right)(-1)^{k+1} \frac{\cos n \theta \sin \theta_{k}^{(n)}}{n\left(\cos \theta-\cos \theta_{k}^{(n)}\right)}$,

$$
x=\cos \theta .
$$

Suppose $f(x)$ to be a continuous function; then it is known that 
the sequence $L_{n}(f), n=1,2, \cdots$, is not convergent ${ }^{1}$ for all $f(x)$. We may even find a continuous function $f_{1}(x)$ such that the sequence $L_{n}\left(f_{1}\right), n=1,2, \cdots$, is divergent for all points of the interval $-1 \leqq x \leqq+1 .^{2}$

Therefore it is interesting to prove the following theorem:

THEOREM. Let $f(x)$ be a continuous function in the interval $-1 \leqq x$ $\leqq+1 ;$ then

(9) $\lim _{n \rightarrow \infty} \frac{1}{2}\left\{L_{n}[f ; \theta-\pi / 2 n]+L_{n}[f ; \theta+\pi / 2 n]\right\}=f(x), \quad x=\cos \theta$,

and the convergence is uniform in the whole interval $-1 \leqq x \leqq+1$.

Between the interpolation polynomials (8) and the partial sums $s_{n-1}(f)$ of the Fourier series of the even function $f(x)$ there is a far reaching analogy. We mention here only the following. On one hand, it is easy to verify that

$$
L_{n}(f)=c_{0}+c_{1} \cos \theta+\cdots+c_{n-1} \cos (n-1) \theta, \quad x=\cos \theta,
$$

where

$$
\begin{aligned}
c_{0}=\frac{1}{n} \sum_{k=1}^{n} f\left(\cos \theta_{k}^{(n)}\right), \quad c_{r}=\frac{2}{n} \sum_{k=1}^{n} f\left(\cos \theta_{k}^{(n)}\right) \cos r \theta_{k}^{(n)}, \\
r=1,2, \cdots, n-1 .
\end{aligned}
$$

On the other hand,

$$
s_{n-1}(f)=a_{0}+a_{1} \cos \theta+\cdots+a_{n-1} \cos (n-1) \theta,
$$

where

$$
\begin{aligned}
& a_{0}=\frac{1}{\pi} \int_{0}^{\pi} f(\cos \theta) d \theta, \quad a_{r}=\frac{2}{\pi} \int_{0}^{\pi} f(\cos \theta) \cos r \theta d \theta, \\
& r=1,2, \cdots, n-1 \text {. }
\end{aligned}
$$

Our theorem is analogous with the well known theorem of Rogosinski in the theory of Fourier series.

We first prove the following lemma.

${ }^{1}$ G. Faber, Über die interpolatorische Darstellung stetiger Funktionen, Jahresbericht der Deutschen Mathematiker-Vereinigung, vol. 23 (1914), pp. 190-210. S. Bernstein, Sur la limitation des valeurs . . . Bulletin de l'Académie des Sciences de l'URSS, 1931, pp. 1025-1050.

${ }^{2}$ G. Grünwald, Über Divergenzerscheinungen der Lagrangeschen Interpolationspolynome stetiger Funktionen, Annals of Mathematics, (2), vol. 37 (1936), pp. 908-918. See also J. Marcinkiewicz, Sur la divergence des polynomes d'intérpolation, Acta Litterarum ac Scientiarum Regiae Universitatis Hungaricae Francisco-Iosephinae, vol. 8 (1937), pp. 131-135. 
LEMMA.

$$
\left.\frac{1}{2} \sum_{k=1}^{n}\left|l_{k}\right| \theta-\pi / 2 n\right]+l_{k}[\theta+\pi / 2 n] \mid<c_{1},
$$

where $c_{1}>0$ is an absolute constant.

From (7) we have, for $\theta \neq \theta_{k}^{(n)} \pm \pi / 2 n$,

$$
\begin{aligned}
& \frac{1}{2}\left(l_{k}[\theta-\pi / 2 n]+l_{k}[\theta+\pi / 2 n]\right) \\
& =\frac{1}{2}(-1)^{k+1}\left(\frac{\cos n(\theta-\pi / 2 n) \sin \theta_{k}^{(n)}}{n\left(\cos (\theta-\pi / 2 n)-\cos \theta_{k}^{(n)}\right)}\right. \\
& \left.\quad+\frac{\cos n(\theta+\pi / 2 n) \sin \theta_{k}^{(n)}}{n\left(\cos (\theta+\pi / 2 n)-\cos \theta_{k}^{(n)}\right)}\right)
\end{aligned}
$$

$$
\begin{aligned}
& =\frac{1}{2}(-1)^{k+1} \frac{\sin \theta_{k}^{(n)} \sin n \theta \sin \theta \sin \pi / 2 n}{4 n \sin \frac{1}{2}\left(\theta+\theta_{k}^{(n)}+\pi / 2 n\right) \sin \frac{1}{2}\left(\theta-\theta_{k}^{(n)}+\pi / 2 n\right)} \\
& \cdot \frac{1}{\sin \frac{1}{2}\left(\theta+\theta_{k}^{(n)}-\pi / 2 n\right) \sin \frac{1}{2}\left(\theta-\theta_{k}^{(n)}-\pi / 2 n\right)}
\end{aligned}
$$

and

$$
\begin{aligned}
& \left|\frac{1}{2}\left(l_{k}[\theta-\pi / 2 n]+l_{k}[\theta+\pi / 2 n]\right)\right| \\
& \quad \leqq \frac{1}{2 n}\left|\frac{\sin \pi / 2 n}{\sin \frac{1}{2}\left(\theta-\theta_{k}^{(n)}+\pi / 2 n\right) \sin \frac{1}{2}\left(\theta-\theta_{k}^{(n)}-\pi / 2 n\right)}\right| \\
& \quad \leqq \frac{1}{2 n} \frac{\pi / 2 n}{(2 / \pi)\left|\frac{1}{2}\left(\theta-\theta_{k}^{(n)}+\pi / 2 n\right)\right|(2 / \pi)\left|\frac{1}{2}\left(\theta-\theta_{k}^{(n)}-\pi / 2 n\right)\right|} \\
& \quad \leqq \frac{\pi^{3}}{4 n^{2}} \frac{1}{\left(\theta-\theta_{k}^{(n)}-\pi / 2 n\right)^{2}} .
\end{aligned}
$$

Now let $\theta$ be fixed and

(16) $1<x_{1}<x_{2}<\cdots<x_{j}<x=\cos \theta<x_{j+1}<\cdots<x_{n}<-1$.

It is known that ${ }^{3}$

$$
\left|l_{k}(x)\right|<4 / \pi, \quad k=1,2, \cdots, n ; n=1,2, \cdots ;-1 \leqq x \leqq+1 .
$$

Thus

${ }^{3}$ P. Erdös and G. Grünwald, Note on an elementary problem of interpolation, this Bulletin, vol. 44 (1938), pp. 515-578. This bound is the best possible; Fejér proved earlier $\left|l_{n}(x)\right|<2^{1 / 2}$. See L. Fejér, Lagrangesche Interpolation und die zugehörigen konjugierten Punkte, Mathematische Annalen, vol. 106 (1932), pp. 1-55. 


$$
\begin{aligned}
\frac{1}{2} & \sum_{k=1}^{n}\left|l_{k}[\theta-\pi / 2 n]+l_{k}[\theta+\pi / 2 n]\right| \\
& \leqq \frac{16}{\pi}+\frac{1}{2} \sum_{1 \leqq k \leqq n, k \neq j-2, j-1, j, j+1}\left|l_{k}[\theta-\pi / 2 n]+l_{k}[\theta+\pi 2 n]\right| \\
& \leqq \frac{16}{\pi}+\sum_{1 \leqq k \leqq n, k \neq j-2, j-1, j, j+1} \frac{\pi^{3}}{4 n_{2}} \frac{1}{\left(\theta-\theta_{\substack{(n) \\
k}}-\pi / 2 n\right)^{2}} \\
& \leqq \frac{16}{\pi}+\frac{\pi^{3}}{4 n^{2}} \sum_{k=1}^{\infty}\left(\frac{2 n}{\pi}\right)^{2} \frac{1}{k^{2}}=c_{1} .
\end{aligned}
$$

If $\theta=0, \pi$, the same inequality evidently holds.

From (17) we obtain for sufficiently large $n, \delta>0$ fixed,

$$
\sum_{1 \leqq k \leqq n,\left|\theta-\theta_{k}^{(n)}\right|>\delta} \frac{1}{2}\left|l_{k}[\theta-\pi / 2 n]+l_{k}[\theta+\pi / 2 n]\right|=O(1 / n) .
$$

Now we are in the position to prove our theorem. Let $\epsilon>0$ be a fixed number. The identity (4) gives

$$
\frac{1}{2} \sum_{k=1}^{n}\left(l_{k}[\theta-\pi / 2 n]+l_{k}[\theta+\pi / 2 n]\right)=1 ;
$$

hence for a fixed $x=\cos \theta$

$$
\begin{aligned}
\frac{1}{2}\left\{L_{n}\right. & {\left.[f ; \theta-\pi / 2 n]+L_{n}[f ; \theta+\pi / 2 n]\right\}-f(\cos \theta) } \\
& =\sum_{k=1}^{n} \frac{1}{2}\left(f\left(\cos \theta_{k}^{(n)}\right)-f(\cos \theta)\right)\left(l_{k}[\theta+\pi / 2 n]+l_{k}[\theta+\pi / 2 n]\right) .
\end{aligned}
$$

The function $f(\cos \theta)$ is continuous; thus we can find a positive number $\delta$ such that

$$
\left|f(\cos \theta)-f\left(\cos \theta_{k}^{(n)}\right)\right|<\epsilon / 2 c_{1}
$$

whenever $\left|\theta-\theta_{k}^{(n)}\right|<\delta$. From (20) and (21) we have

$$
\begin{aligned}
& \Delta=\left|\frac{1}{2}\left\{L_{n}[f ; \theta-\pi / 2 n]+L_{n}[f ; \theta+\pi / 2 n]\right\}-f(\cos \theta)\right| \\
& =\mid \sum_{1 \leqq k \leqq n, \mid \theta-\theta_{k}^{(n)}<\delta} \frac{1}{2}\left(f\left(\cos \theta_{k}^{(n)}\right)-f(\cos \theta)\right)\left(l_{k}[\theta-\pi / 2 n]+l_{k}[\theta+\pi / 2 n]\right) \\
& +\sum_{1 \leqq k \leqq n,\left|\theta-\theta_{k}^{(n)}\right|>\delta} \frac{1}{2}\left(f\left(\cos \theta_{k}^{(n)}\right)-f(\cos \theta)\right)\left(l_{k}[\theta-\pi / 2 n]+l_{k}[\theta+\pi / 2 n]\right) \\
& \leqq \frac{\epsilon}{2 c_{1}} \sum_{1 \leqq k \leqq n,\left|\theta-\theta_{k}^{(n)}\right|<\delta} \frac{1}{2}\left|l_{k}[\theta-\pi / 2 n]+l_{k}[\theta+\pi / 2 n]\right| \\
& +\sum_{1 \leqq k \leqq n,\left|\theta-\theta_{k}^{(n)}\right|>\delta} \frac{1}{2}\left|f\left(\cos \theta_{k}^{(n)}\right)-f(\cos \theta)\right|\left|l_{k}[\theta-\pi / 2 n]+l_{k}[\theta+\pi / 2 n]\right|
\end{aligned}
$$


and by the lemma and (18) for sufficiently large $n$

$$
\begin{aligned}
\Delta & \leqq \frac{\epsilon}{2 c_{1}} c_{1}+\sum_{1 \leqq k \leqq n,\left|\theta-\theta_{k}^{(n)}\right|>\delta} \frac{1}{2} 2 M\left|l_{k}[\theta-\pi / 2 n]+l_{k}[\theta+\pi / 2 n]\right| \\
& <\epsilon / 2+M O(1 / n)<\epsilon,
\end{aligned}
$$

where $M=\max _{-1 \leqq x \leqq+1}|f(x)|$, and this proves our theorem.

Budapest, Hungary

\section{DISCONTINUOUS CONVEX SOLUTIONS OF DIFFERENCE EQUATIONS ${ }^{1}$}

\section{FRITZ JOHN}

This paper contains some conditions for continuity of convex solutions of a difference equation.

A function $f(x)$ defined for $a \leqq x \leqq b$ is convex, if

$$
\left(\frac{x+y}{2}\right) \leqq \frac{f(x)+f(y)}{2} .
$$

If $f(x)$ is convex and bounded from above in $a \leqq x \leqq b$, then $f(x)$ is continuous (see Bernstein [1, p. 422]). ${ }^{2}$ If $f(x)$ is convex in $a \leqq x \leqq b$ and $y$ a fixed number with $a<y<b$, let the function $\phi_{y}(x)$ be defined by

$$
\phi_{y}(x)=\lim _{\alpha \rightarrow x-y} f(y+\alpha),
$$

where $\alpha$ assumes rational values only. Then $\phi_{y}(x)$ is uniquely defined, continuous, and convex for $a<x<b$ (F. Bernstein [1, p. 431, Theorem 7]); moreover $\phi_{y}(x)=f(x)$ for rational $y-x$.

THEOREM 1. If there exists at most one continuous convex solution of the difference equation

$$
F(x, f(x), f(x+1), \cdots, f(x+n))=g(x), \quad x>0,
$$

where $F$ and $g$ are continuous functions of their arguments, then there exist no discontinuous convex solutions.

Proof. If $f(x)$ is a convex solution, then, for $x-y$ rational,

$$
F\left(x, \phi_{y}(x), \phi_{y}(x+1), \cdots, \phi_{y}(x+n)\right)=g(x) ;
$$

\footnotetext{
1 Presented to the Society, September 12, 1940.

2 The numbers in brackets refer to the bibliography.
} 